\title{
PLASMA HEATING BY THE PARAMETRIC EXCITATION OF ACOUSTIC WAVES IN CORONAL MAGNETIC LOOPS
}

\author{
V. V. Zaitsev ${ }^{\ddagger}$, and K. G. Kislyakova ${ }^{\S}$
}

\begin{abstract}
When studying microwave emission of active regions on the Sun, an effect of parametric resonance between 5-min velocity oscillations in the solar photosphere and sound oscillations of coronal magnetic loops modulating the microwave emission has been discovered for the first time. The effect shows itself as simultaneous excitation in coronal magnetic loop oscillations with periods 5,10 , and $3 \mathrm{~min}$, which correspond to the pumping frequency, subharmonic, and the first upper frequency of parametric resonance. The parametric resonance can serve as an effective channel of transporting the energy of photospheric oscillations into the upper layers of the solar atmosphere. The energy of acoustic waves excited in a coronal magnetic loop, rate of dissipation of acoustic waves, and rate of heating of the coronal plasma are determined. The maximum temperature predicted for the apex of the loop is calculated as a function of velocity of photospheric oscillations, length of the loop, and electric current in the loop. It is shown that the mechanism proposed can explain the origin of quasi-stationary X-ray loops with temperatures of 3-6 MK. The lengths of these loops are resonant for acoustic waves excited by the 5 -min photospheric oscillations. The use of the proposed mechanism to explain heating of the X-ray loops expected to be on stars of late spectral types is discussed.
\end{abstract}

\section{Introduction}

Identification of the energy source that heats the plasma of the solar and stellar coronas is important for coronal physics. Many possible energy sources which could work in the coronal magnetic loops are suggested. For example, considering heating by currents estimations of necessarily currents values in a coronal magnetic loop can be made. In this case currents exceeding the value of $10^{15} \mathrm{~A}$ are demanded, while the observed values

\footnotetext{
$\bar{\ddagger}$ Institute of Applied Physics, Russian Academy of Science, 46 Ul'yanov Street, 603950, Nizhni Novgorod, Russia

$\S$ Lobachevsky State University of Nizhni Novgorod, 23 Prospekt Gagarina, 603950, Nizhni Novgorod, Russia
} 
are in the order of $10^{10}-10^{11} \mathrm{~A}$ [Zaitsev and Kruglov, 2009; Haguard, 1988]. Tearing instability, reconnection and microflares could be considered as a second possible source of coronal plasma heating. Typical energy release during a separate microflare can be calculated, $P=10^{24}-10^{25} \mathrm{erg} \times \mathrm{s}^{-1}$. Comparing it with the energy required to explain coronal temperatures, $10^{26} \mathrm{erg} \times \mathrm{s}^{-1}$, the required microflares occurrence rate is $10-100$ s. But in the reality the microflares are not obligatory observed every hundred seconds. Photospheric convection capable of exciting waves in coronal magnetic loops, with the dissipation of these waves being able to heat the coronal plasma, is among the most effective energy sources. The use of such mechanisms in solar physics was studied by Ionson [1982], and for red dwarfs by Mullan and Johnson [1995]. But when we consider the photospheric convection as an energy source for coronal plasma heating we need the additional mechanism which could explain how do this energy penetrate into the corona and coronal magnetic loops. The efficient excitation of waves in coronal magnetic loops requires a coincidence of the period of the global mode of the loop with the characteristic time scale of the photospheric convection. There are the so-called five-minute oscillations in the velocity of the photospheric convection, associated with global oscillations of the solar gaseous sphere. The periods of these oscillations are $\tau_{5} \approx 100-400 \mathrm{~s}$, with the spectrum maximum being at $300 \mathrm{~s}$. In the magnetic loops of the solar corona, the global Alfvenic mode has periods $P_{A} \approx 0.5-5 \mathrm{~s}$ [Nitta et al., 1991]. Therefore, photospheric convection is incapable of exciting Alfven waves. Also it is to mention that dissipation of the Alfven waves is too small to compensate the radiation loss. On the other hand, the slow magnetoacoustic waves in coronal magnetic loops have periods which coincide with the time scales of the photospheric convection. Therefore, acoustic waves can be efficiently excited in coronal magnetic loops by photospheric convection, with its energy being transformed into the thermal energy of the coronal plasma via the dissipation of the acoustic waves.

\section{Parametric Resonance}

The excitation of acoustic waves in current carrying coronal magnetic loops by a parametric resonance with the five-minute oscillations in the velocity of the photospheric convection was studied in Zaitsev et al. [2006] and Zaitsev et al. [2008]. Let us note some conclusions of this work. Let us suppose that the velocity of the photospheric convection oscillates near its mean (for example, due to the five-minute photospheric oscillations) following the law $\left|V_{r}\right|=V_{0}+V_{\sim} \sin \omega t$ where $V_{\sim}<<V_{0}$ near the mean value $V_{0}$. The current flowing along the loop is $I_{z}=I_{0}+I_{\sim}$, where $I_{\sim}$ and $I_{0}$ are variable and constant components of the electric current flowing along the loop. The relative amplitude of the currents components takes the form $\frac{I_{\sim}}{I_{0}} \approx \frac{h V_{\sim}}{\omega L r_{0}}$, where $h=500-100 \mathrm{~km}$ is the height interval involving the electromotive force, $r_{0}$ the radius of the magnetic tube at its bases, and $L$ the inductance of the loop as an equivalent electric circuit [Landau and Lifschits, 1984]. It occurs due to following dependence between the electromotive force and the radial velocity: $\epsilon=\frac{V_{r} B_{\phi} h}{c}$. The tube equilibrium condition for the radial variable then 
results in a periodically varying tube pressure with the amplitude

$$
p_{\sim}=\frac{4}{3} \frac{I_{0} I_{\sim}}{\pi c^{2} r^{2}}
$$

where $c$ is the speed of light and $r$ the tube radius, which is assumed to be constant in the corona. Consequently, the sound speed acquires a periodic modulation with the period of the five-minute oscillations, and the equation for the acoustic waves takes the form

$$
\frac{\partial^{2} V_{z}}{\partial t^{2}}+\omega_{0}^{2}(1+q \cos \omega t) V_{z}=0
$$

where

$$
\omega_{0}^{2}=k_{\|}^{2} c_{s 0}^{2}, \quad k_{\|}=\frac{s \pi}{l}, \quad s=1,2,3, \ldots, \quad q=\frac{4}{3} \frac{\gamma-1}{\gamma} \frac{I_{0} I_{\sim}}{\pi c^{2} r^{2} p_{0}} .
$$

In (3), $c_{s 0}=\left(\gamma k_{B} T_{0} / m_{i}\right)^{1 / 2}$ is the sound speed, $p_{0}=2 n k_{B} T_{0}$ the pressure, $T_{0}$ the unperturbed temperature, and $\gamma=c_{p} / c_{v}$ the ratio of specific heats. Expression (2) is the Mathieu equation describing the parametric instability (see, for example, Landau and Lifschits [1988]). Here $q$ is a coefficient determining the width of parametric instability zones near frequencies of a parametric resonance $\omega_{n}=\frac{n \omega}{2}, \quad n=1,2,3, \ldots$. This indicates that if the coronal magnetic loop is exposed to the five-minute photospheric oscillations, the excitation of acoustic waves with periods of 10, 5, 3.3 min etc. is possible in the loop. However, the excitation occurs only when the eigenfrequency of the acoustic oscillations of the loop $\omega_{0}$ is retained within the first zone of instability that approaches $\omega / 2$. The width of this zone is of the order of $q$, namely $-\frac{q \omega_{0}}{2}<\frac{\omega}{2}<\frac{q \omega_{0}}{2}$. This means that the coronal magnetic loop must have an appropriate length for the five-minute photospheric oscillations to excite the acoustic waves there.

\section{Energy of the Acoustic Waves}

The part played by the acoustic waves in coronal plasma heating is considered in Zaitsev and Kislyakova [2010]. Let us note some conclusions from this paper. The velocity amplitude $\mathrm{v}$ of the acoustic waves is expressed through the pressure amplitude $p_{\sim}$ by the relation $\nu=p_{\sim} / \rho_{0} c_{s 0}$, where $\rho_{0}$ is the unperturbed plasma density. Keeping the relation (1) in mind, the following formula for the mean energy density of the acoustic waves can be obtained:

$$
W_{s}=\frac{\rho_{0} v^{2}}{2}=\frac{16}{18} \frac{1}{\rho_{0} c_{s 0}^{2}}\left(\frac{I_{0}^{2}}{\pi c^{2} r^{2}}\right)^{2}\left(\frac{I_{\sim}}{I_{0}}\right)^{2} .
$$

Hence, the energy density depends on the amplitude of the electric current, modulated by the five-minute oscillations in the velocity of the photospheric convection. We can determine the amplitude $I_{\sim}$ by analyzing the low-frequency modulation of the microwaves radiated by the coronal magnetic loops [Zaitsev et al., 2008]. A narrow-band modulation whose frequency is a fraction of a Hertz, which corresponds to free oscillations of the loop as an equivalent electric circuit, is frequently observed in the microwave radiation of flares. In this case, the frequency of the oscillations depends on the electric current in the circuit through the self-consistent magnetic field, while a high inductance in the loop 


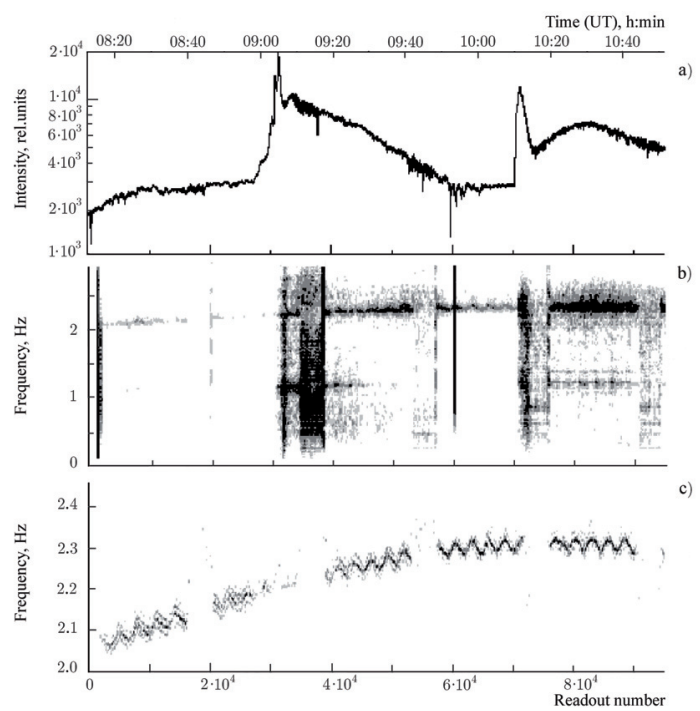

Figure 1: Temporal profile and dynamic spectrum of the low-frequency modulation of the microwave intensity observed at $37 \mathrm{GHz}$, obtained using Fourier transform [Zaitsev et al., 2008]

provides a high Q factor for the oscillations. For fairly high currents flowing in the loop $\left(I>\operatorname{cr} B_{z}(0) / 2\right.$, where $B_{z}(0)$ is the magnetic field on the loop axis) the eigenfrequency of the equivalent electric circuit is proportional to the current [Zaitsev and Stepanov, 2008],

$$
\nu_{R L C} \approx \frac{I}{2 \pi c r^{2} \sqrt{2 \pi \Lambda n_{0} m_{i}}}, \quad \Lambda=\ln \frac{4 l}{\pi r}-\frac{7}{4},
$$

where $l$ is the length of the loop. Therefore, when the five-minute photospheric oscillations modulate the electric current in the loop, the eigenfrequency of the equivalent circuit is also modulated by the five-minute period, and the relative depth of the frequency modulation coincides with the relative depth of the current. An example of such a modulation is presented in Zaitsev et al. [2008].

The figure in Zaitsev et al. [2008] shows (a) the temporal profile of the intensity of the $37 \mathrm{GHz}$ microwaves, (b) the dynamic spectrum of the low-frequency modulation of the radio intensity obtained via a fast Fourier transform with a sliding window, and (c) the same dynamic spectrum obtained using the Wigner-Ville method for two microwave bursts observed on August 28, 1990. The last transform exhibits a considerably higher frequency-time resolution, and the fiveminute modulation is clearer there. Plot (c) shows that the relative depth of the frequency modulation $\nu_{L R C}$ is of the order of a few percent. Similar results were found for a number of similar events [Zaitsev et al., 2008]. For further estimates, we can assume

$$
\frac{I_{\sim}}{I_{0}} \approx \frac{\Delta \nu_{R L C}}{\nu_{R L C}} \approx 10^{-2} .
$$


Expressions (4) and (6) show that the energy density of the excited acoustic waves can be comparable with the thermal energy of the plasma for fairly high electric currents flowing in the coronal magnetic loops.

\section{Dissipation of the Acoustic Waves or the Heating Rate}

Dissipative effects, such as viscosity and electrical and thermal conductivity, transform the energy of acoustic waves into heat. When the damping decrement $\gamma_{s}$ describing the dissipation of the energy of acoustic waves is much lower than the frequency, $\gamma<<\omega$, the energy transformed into heat per unit time and unit volume, or the heating rate, is $H=\gamma_{s} W_{s}$. For the case of acoustic waves, the damping decrement takes the form [Braginskii, 1963] $\gamma_{s}=\gamma_{J}+\gamma_{v}+\gamma_{T}$, where the terms due to the electric conductivity, viscosity, and thermal conductivity are

$$
\gamma_{J}=\frac{c}{4 \pi \sigma} \frac{c_{s}^{2}}{c_{A}^{2}} k, \quad \gamma_{V}=0.18 \omega^{2} \tau_{i}, \quad \gamma_{T}=0.12 \frac{m_{i}}{m_{e}} \omega^{2} \tau_{e},
$$

where $\sigma$ is the electric conductivity, $c_{A}$ the Alfven speed, and $\tau_{i, e}$ the characteristic times for ion-ion and electro-ion collisions, respectively. Estimates of terms contained in (7) show that the electron thermal conductivity makes the main contribution to the ion acoustic dissipation, described by the damping decrement $\gamma_{T} \sim 7 \times 10^{-2} \omega$, (for $n \approx 10^{9} \mathrm{~cm}^{-3}$, $T \approx 10^{6} \mathrm{~K}$ and $\omega \approx 0.02 \mathrm{c}^{-1}$ ). Making use of (5) and (7), the following relation can be obtained for the heating rate:

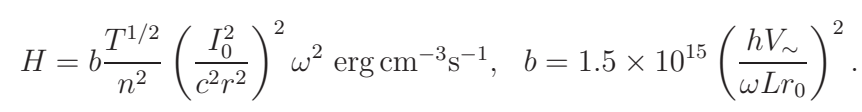

The necessary condition for heating is that the heating rate must exceed the radiation losses, $H \geq H_{R}=n^{2} \chi(T)$. The losses are maximum when $\chi(T)=10^{-21.2}$ at $T \approx 10^{6} \mathrm{~K}$ [Rosner, 1978]. Supposing that $n \approx 10^{9} \mathrm{~cm}^{-3}, T \approx 10^{6} \mathrm{~K}, \omega \approx 2 \times 10^{-2} \mathrm{~s}^{-1}$ (which corresponds to the five-minute oscillation period), we obtain the heating condition $B_{\phi} \approx$ $\left(I_{0} / \mathrm{cr}\right) \geq 10 \mathrm{G}$. Here, $B_{\phi}$ is the non-potential portion of the magnetic field due to the current flowing along the coronal magnetic loop, which corresponds to weak twistedness of the magnetic field observed in loopswith magnetic fields of $10^{2}-10^{3} \mathrm{G}$. This estimate for $B_{\phi}$ corresponds to an electric current $I_{0} \geq 10^{10}$ A flowing through the transverse section of the loop. Thus, the parametric resonance becomes an efficient source of the plasma heating when the electric current in the loop exceeds some critical value. The second necessary condition is that the loop must have an appropriate length, $l \approx \frac{2 \pi c_{s 0}}{\omega} \mathrm{cm}$. Typical frequencies of the five-minute oscillations in the photospheric velocity are in the interval $1.5 \times 10^{-2} \mathrm{~s}^{-1}<\omega<6 \times 10^{-2} \mathrm{~s}^{-1}$ with the spectral maximum being at the frequency $\omega \approx 2 \times 10^{-2} \mathrm{~s}^{-1}$. Therefore, coronal magnetic loops with lengths in the interval $2 \times 10^{9} \leq l \leq 2 \times 10^{10} \mathrm{~cm}$ will be "resonant". It is worth noting that the "warm" magnetic loops with temperatures $\sim 1.5 \times 10^{6} \mathrm{~K}$ observed by TRACE [Aschwanden et al., 1999] have lengths $l \approx(1.5-7) \times 10^{10} \mathrm{~cm}$, which are outside the "resonant" interval, while the "hot" X-ray loops with temperatures $\sim(3-6) \times 10^{6} \mathrm{~K}$ observed by Yohkoh [Kano and Tsuneta, 1995] display lengths of $l \approx(0.2-3) \times 10^{10} \mathrm{~cm}$ which fall into the "resonant" interval. 
This may indicate that the parametric resonance plays an important role in the origin of hot stationary X-ray loops, which lenghts fall into the resonant interval. It should be noticed, that the flare loops in general have lenghts in the range $5 \times 10^{8} \leq l \leq 5 \times 10^{9} \mathrm{~cm}$, which are not "resonant", so this type of loops should possess another heating source. In the current paper we concentrate on the possible heating mechanism of the stationary X-ray loops.

\section{Heating of Coronal Magnetic Loops}

The heat balance equation for the stationary tube takes the form [Priest, 1982]

$$
\frac{\partial}{\partial s} k_{e} T^{5 / 2} \frac{\partial T}{\partial s}=n^{2} \chi(T)-H
$$

where $k_{e}=0.92 \times 10^{-6} \mathrm{erg} \mathrm{s}^{-1} \mathrm{~K}^{-7 / 2}, \chi(T)=\chi_{0} T^{-1 / 2}$. We measure the coordinate $s$ along the loop from the chromosphere temperature minimum. In addition, we allow for the symmetry at the apex of the loop. The boundary conditions for (9) then take the form

$$
\frac{d T}{d s}=0 \quad\left(s=0, \quad T=T_{0}\right), \quad \frac{d T}{d s}=0 \quad\left(s=\frac{l}{2}, \quad T=T_{1}\right) .
$$

Solving the heat balance equation (9) and taking into account the boundary conditions [Priest, 1982] the well-known equation can be obtained, determining the relation between the pressure, length of the loops and temperature in the apex of the loop:

$$
l p=A\left(\frac{8 k_{B}^{2} k_{e}}{\chi_{0}}\right)^{1 / 2} T_{1}^{3}, \quad A=\int_{0}^{1} x^{2}\left(1-x^{5}\right)^{-1 / 2} d x \approx 0.55 .
$$

The temperature at the loop apex as a function of the length can also be determined:

$$
T_{1}=\left(\frac{b \chi_{0} \omega^{2}}{24 k_{e}^{2} A^{4}}\right)^{1 / 7}\left(B_{\phi} l\right)^{4 / 7}=9.5\left(\frac{h V_{\sim}}{L r_{0}}\right)^{2 / 7} \approx 7\left(\frac{h V_{\sim} l B_{\phi}^{2}}{r_{0}}\right)^{2 / 7},
$$

where $B_{\phi} \approx I_{0} / \mathrm{cr}$ is the mean azimuthal component of the magnetic field in the coronal magnetic loop. Formula (12) shows that the temperature in the loop increases with the loop length, the electric current flowing along the loop, and the velocity of the photospheric oscillations. Finally, the maximum temperature is independent of the frequency of the photospheric oscillations, since the coefficient $b$ in (12) decreases as the frequency squared [see (12)]. For $l=10^{10} \mathrm{~cm}$ (the typical lengths of hot X-ray loops), (12) yields the estimate for the typical temperature of the hot X-ray loops observed by Yohkoh $T_{1}=(4-6) \times 10^{6} \mathrm{~K}$, if we assume an azimuthal magnetic field $B_{\phi}=5-10 \mathrm{G}$, which corresponds to an electric current $5 \times 10^{9}-10^{10}$ A flowing through the transverse section of the coronal magnetic loop. 


\section{Discussion}

\subsection{Magnetic Loops in the Solar Corona}

For fairly high electric currents flowing in coronal magnetic loops, the energy density of the excited acoustic waves can be comparable with the thermal energy density of the plasma. An important feature of the acoustic waves (compared, for example, with Alfven waves) is their efficient dissipation. Quasi-stationary X-ray loops in the solar corona were studied using the Yohkoh soft X-ray telescope [Kano and Tsuneta, 1995]. These loops were distinguished from similar non-stationary objects in both their pressure and temperature, and a specific heating mechanism was supposed there. Analysis of these data yields the dependence of the temperature on the loop length in the range $T \propto l^{0.27-0.52}$. We have shown that the dissipation of ion-acoustic waves excited by the photospheric five-minute oscillations in the presence of a parametric resonance with the free acoustic oscillations of the coronal magnetic loops is a possible heating mechanism. This mechanism exhibits a similar dependence of the temperature on the length, namely $T \propto l^{0.286}$ [see (12)].

\subsection{Magnetic Loops on Late-type Stars}

It is generally accepted that the coronas of active stars are filled with magnetic loops, quite similar to the solar corona. This is supported, in particular, by observations of pulsating optical and X-ray emission from active stars, associated with oscillations of magnetic loops in the stellar coronas [Mullan and Johnson, 1995]. Such pulsations have been observed both during and outside periods of flare activity. Soft X-ray oscillations which have periods $\mathrm{P}$ ranging from several tens to several hundreds of seconds and are not associated with any flares have been observed for several dMe stars [Mullan and Johnson, 1995]. The typical lengths and temperatures of coronal magnetic loops have been determined for these stars [Mullan et al., 2006] (see the table I). These pulsations can probably be excited by photospheric convection in the presence of parametric resonance between the oscillations in the velocity of the photospheric plasma and the free acoustic oscillations of magnetic loops.

Table 1: Typical lengths and temperatures of coronal magnetic loops

\begin{tabular}{|c|c|c|c|c|}
\hline Star & $R, R_{\text {Sun }}$ & $l, 10^{9} \mathrm{~cm}$ & $T, 10^{6} \mathrm{~K}$ & $\mathrm{P}, s$ \\
\hline AD Leo & 0.35 & $4-40$ & $2-3$ & $150-190$ \\
Proxima Cen & 0.145 & $0.5-9$ & $1-3$ & $66-68$ \\
UV Cet & 0.15 & $0.7-2$ & $2-3$ & 56 \\
\hline
\end{tabular}

\section{Conclusions}

Finally, the following main conclusions of our work can be mentioned: - The parametric resonance can serve as an effective channel of transporting the energy of photospheric 
oscillations into the upper layers of the solar atmosphere. This effect opens up the important prospects in understanding the mechanisms of coronal plasma heating. - The proposed mechanism can explain the origin of quasi-stationary X-ray loops with temperatures of 3-6 MK. The lengths of these loops are resonant for acoustic waves excited by the 5-min photospheric oscillations. - Proposed mechanism can also be used to the X-ray loops heating on stars of late spectral classes.

Acknowledgements. This work was partially supported by the Russian Foundation for Basic Research (project no. 11-02-00103-a), by the project NK-21P of Education Ministry of Russian Federation and by the EU grant \#228319 in the framework of the Project Europlanet RI-FP7.

\section{References}

Aschwanden, M. J., J. S. Newmark, J.-P. Delaboudinière, W. M. Neupert, J. A. Klimchuk, G. A. Gary, F. Portier-Fozzani, A. Zucker, Three-dimensional stereoscopic analysis of Solar active region loops. I. SOHO/EIT observations at temperatures of (1.0-1.5) x $10^{6}$ K, Astrophys. J., 515, 842-867, 1999.

Braginskii, S. I., Transport phenomena in plasma, in Plasma Theory Questions, Vol. 1 [in Russian], edited by M. A. Leontovich, Gosatomizdat, Moscow, 1963.

Hagyard, M. J., Observed nonpotential magnetic fields and the inferred flow of electric currents at a location of repeated flaring, Solar Phys., 115, 107-114, 1988.

Ionson, J. A., Resonant electrodynamic heating of stellar coronal loops - an LRC circuit analog, Astrophys. J., 254, 318-334, 1982.

Kano, R., and S. Tsuneta, Scaling law of solar coronal Loops obtained with YOHKOH, Astrophys. J., 454, 934, 1995.

Landau, L.D., and E.M. Lifschits, Course of Theoretical Physics, Vol. 1: Mechanics, Nauka, Moscow, 1982.

Landau, L. D., and E. M. Lifschits, Course of Theoretical Physics, Vol. 8: Electrodynamics of Continuous Media, Nauka, Moscow, 1982.

Mullan, D. J., and M. Johnson, Coronal heating in flare stars: resonant MHD absorption? Astrophys. J., 444, 350-362, 1995.

Mullan, D. J., M. Mathiondakis, D. S. Bloomfield, and D. J. Christian, A comparative study of flaring loops in active stars, Astrophys. J. Suppl. Ser., 164, 173-201, 2006.

Nitta, N., S. M. White, M.R. Kundu, N. Gopalswamy, G. D. Holman, J. W. Brosius, J. T. Schmelz, J. L. R. Saba, and K. T. Strong, Coronal magnetic structures observing campaign. I - Simultaneous microwave and soft x-ray observations of active regions at the Solar limb, Astrophys. J., 374, 374-385, 1991.

Priest, E. R., Solar Magnetohydrodynamics, Reidel, Dordrecht, 1982. 
Rosner, R., W.H. Tucker, and G.S. Vaiana, Dynamics of the Quiescent Solar Corona, Astrophys. J., 230, 643-645, 1978.

Zaitsev, V.V., and Kislyakov A. G., Parametric excitation of acoustic oscillations in closed coronal magnetic loops, Astron. Rep., 50, 823-833, 2006.

Zaitsev, V. V., and A. V. Stepanov, Reviews of Topical Problems: Coronal magnetic loops, Phys. Usp., 51, 1123-1160, 2008.

Zaitsev, V. V., and A. A. Kruglov, Electrical currents in coronal magnetic loops, Radiophys. Quant. Electr., 52, 5-6, 2009.

Zaitsev, V.V. and K. G. Kislyakova, Plasma heating during the parametric excitation of acoustic waves in coronal magnetic loops, Astron. Rep., 54, 367-373, 2010.

Zaitsev, V.V., A. G. Kislyakov, and S. Urpo, Manifestations of the 5-min photospheric oscillations in the solar microwave emission, Radiophys. Quantum Electron., 46, 893-903, 2003.

Zaitsev, V.V., A. G. Kislyakov, and K. G. Kislyakova, Parametric resonance in the solar corona, Cosmic Res., 46, 301-308, 2008. 
